\section{NSCLC: Für wen Pemetrexed plus Carboplatin?}

\begin{abstract}
Für Patienten mit Rezidiv eines nichtkleinzelligen Lungenkarzinoms (NSCLC) ist die Prognose ungünstig. In einer aktuellen Studie wurde ein VoraussageModell entwickelt, um für individuelle Patienten mit Nichtplattenepithel (nicht-SCC)-NSCLC vor Zweitlinienbeginn den Therapieeffekt von Pemetrexed plus Carboplatin gegenüber Pemetrexed abzuschätzen.
\end{abstract}

\begin{abstract}
n einer kombinierten Analyse zweier randomisierter Studien (NVALT-7, GOIRC 02-2006) hatte sich kein Vorteil für Pemetrexed plus Carboplatin (PC) versus Pemetrexed (P) in der Zweitlinientherapie des Nicht-SCC-NSCLC gezeigt. Weil einzelne Patienten dennoch profitieren könnten, wurde ein Voraussage-Modell erarbeitet. Zur Modellentwicklung wurden Daten der NVALT-7-Studie genutzt $(\mathrm{n}=180)$, zur Validierung Daten der GOIRC-02-2006-Studie $(\mathrm{n}=210)$.

Mit vordefinierten Patienten- und Tumorcharakteristika wurde ein sogenanntes Weibull-Modell erstellt, mit dem sich der Gewinn der kombinierten Chemotherapie für das mediane progressionsfreie Überleben (PFS) ermit-
\end{abstract}

teln lässt. Darin zeigte sich eine große Spannbreite bezüglich der Effekte von PC gegenüber P auf das PFS - im Median betrug der Anstieg 0,7 Monate (Interquartilsabstand: -0,1-1,5 Monate).

Am meisten profitierten Frauen, Patienten im Stadium IV, Betroffene mit hohem Body-Mass-Index und/oder Adenokarzinom. In einer Entscheidungskurven-Analyse wurde bestätigt, dass das Modell Patienten, die von der Kombination profitieren, adäquat identifiziert. Aktualisierte Nachbeobachtungsdaten zeigten, dass man auch mit einer gewissen Verbesserung des Gesamtüberlebens rechnen darf.

Im Gegensatz zur Subgruppenanalyse, die sich auf Einzelfaktoren konzentriert, erfasst das Weibull-Modell eine Vielzahl unterschiedlicher Parameter parallel, was eine genauere Voraussage ermöglicht. $\mathrm{Zu}$ beachten ist, dass das Modell anhand zweier Studien erstellt wurde, die spezifische Aufnahmekriterien hatten, wie z. B. guter ECOG-PerformanceStatus, befriedigende Organfunktion etc. Die Ergebnisse sind daher nicht ohne weiteres auf andere Patientengruppen übertragbar.

Fazit: Bei vorbehandelten Patienten mit fortgeschrittenem Nicht-SCC-NSCLC sind die Effekte von Pemetrexed-Carboplatin versus Pemetrexed auf das PFS sehr heterogen. Der Therapieeffekt kann im Voraus anhand eines Modells geschätzt werden, das sich auf routinemäßig erhobene Patienten- und Tumorparameter stützt.

Brigitte Schalhorn

van Kruijsdijk RC et al. Pemetrexed plus carboplatin versus pemetrexed in pretreated patients with advanced non-squamous non-small cell lung cancer: treating the right patients based on individualized treatment effect prediction. Ann Oncol. 2016;27(7):1280-6.

\title{
TLI - prognostische Bedeutung beim resektablen NSCLC
}

Die prognostische Relevanz der Tumorinfiltration durch Lymphozyten (TLI) wurde bereits für eine Reihe unterschiedlicher Tumoren nachgewiesen. In einer großen Studie wurde der prognostische Wert der TLI bei Patienten mit reseziertem nichtkleinzelligem Lungenkarzinom (NSCLC) geprüft.

$\mathrm{D}$ ie Forscher analysierten Daten von 4 Studien, in denen der Effekt einer platinbasierten adjuvanten Chemotherapie gegenüber Beobachtung untersucht wurde. Einen Teil der Daten nutzten sie zur Erkundung des Zusammenhangs von TLI und Prognose (sog. Discovery-Set), den anderen zur Validierung (sog. Validation-Set). Grundlage für das Discovery-Set war die IALT-Studie $(n=824)$, für das Validation-Set 3 weitere Studien ( $\mathrm{n}=984$; ANITA, JBR 10, CALGB 9633). Die TLI-Intensität wurde eingeteilt in intensiv versus nichtintensiv.

Komplett auswertbar waren 783 (Discovery-Set) bzw. 763 Patienten (Validation-Set). Das mediane Follow-up betrug 4,8 bzw. 6 Jahre. Die TLI war inten- siv bei $11 \%$ der Patienten im Discoveryund $6 \%$ im Validation-Set $(p<0,001)$. Der prognostische Wert der TLI für den primären Endpunkt Gesamtüberleben (OS) erwies sich in beiden Sets als signifikant, mit einer Hazard Ratio (HR) von $0,56$ ( $p=0,002)$ bzw. 0,45 ( $\mathrm{p}=0,01)$. Ähnliches galt für das krankheitsfreie und spezifisch krankheitsfreie Überleben. Es ließ sich keine statistisch signifikante Heterogenität zwischen den Studien feststellen ( $p \geq 0,38$ für alle Endpunkte).

Für eine explorative Analyse wurden beide Sets kombiniert. Hier ergab sich eine grenzwertig signifikante Interaktion zwischen TLI und Histologie im Hinblick auf das OS mit einer HR von 0,62 für das
Plattenepithelkarzinom, 0,69 für das Adenokarzinom sowie 0,12 für andere Formen des NSCLC. Im Gegensatz zu Ergebnissen aus Brustkrebsstudien zeigte sich in dieser Studie kein signifikanter prädiktiver Effekt für TLI, d.h. es fand sich keine signifikante Interaktion zwischen TLI und der Art der Behandlung in Bezug auf das Überleben.

Fazit: Eine intensive TLI wurde nur bei einer Minderheit der untersuchten NSCLC gefunden. Sie konnte als unabhängiger, günstiger prognostischer Marker für das Überleben bei komplett reseziertem NSCLC validiert werden. Eine starke TLI ist jedoch kein prädiktiver Marker für ein besonders gutes oder schlechtes Ansprechen auf die adjuvante Chemotherapie.

Brigitte Schalhorn

Brambilla E et al. Prognostic effect of tumor lymphocytic infiltration in resectable nonsmall-cell lung cancer. J Clin Oncol. 2016; 34(11):1223-30. 